\title{
AVALIAÇÃO DE RISCOS À SAÚDE DE TRABALHADORES EXPOSTOS A BAIXAS TEMPERATURAS EM ABATEDOURO DE FRANGOS
}

\section{EVALUATION OF REALTH RISKS OF WORKERS EXPOSED TO LOW TEMPERATURES IN BROILER SLAUGHTERHOUSE}

\author{
Fabiano Takeda* E-mail: takeda.f@bol.com.br \\ Antonio Renato Pereira Moro*E-mail: renato.moro@ufsc.com.br \\ Saulo Guths* E-mail: saulo@Impt.ufsc.br \\ *Universidade Federal de Santa Catarina (UFSC), Florianópolis, SC
}

\begin{abstract}
Resumo: O objetivo desse estudo foi analisar a exposição a baixas temperaturas em atividades de cortes de frangos e verificar se há exposição a riscos à saúde ocupacional de acordo com os resultados da avaliação conforme critérios normativos da Organização Internacional de Normalização (ISO) e dos resultados da avaliação utilizando um sistema de monitoramento de temperatura corporal. Para mensurar os dados foram realizadas avaliações quantitativas de temperatura em sítios corporais específicos da mão e pé de trabalhadores e também foi realizada avaliação ambiental nos postos de trabalho. Os resultados mostraram que apesar da atividade representar um PMV adequado, conforme critérios da ISO, os resultados do sistema de monitoramento de temperatura corporal apresentam condições de frio e dor no dedo da mão e no hálux durante quase toda jornada de trabalho. Conclui-se que é arriscado afirmar que condições de exposição a baixas temperaturas podem ser percebidas como confortáveis e sem exposição a riscos à saúde quando avaliadas utilizando apenas os parâmetros estabelecidos nas normas ISO.
\end{abstract}

Palavras-chave: Saúde do trabalhador. Frio. ISO 7730.

Abstract: The objective of this study was to analyze the exposure to low temperatures in broiler cutting activities and to verify if there is exposure to occupational health risks according to the results of the evaluation according to normative criteria of the International Organization for Standardization (ISO) and the results of the evaluation using a body temperature monitoring system. In order to measure the data, quantitative temperature assessments were carried out on specific body sites of workers' hands and feet, and an environmental assessment was also carried out in the work stations. The results showed that although the activity represents an adequate PMV, according to ISO criteria, the results of the body temperature monitoring system show conditions of cold and pain in the finger and the hallux during almost every working day. It is concluded that it is risky to say that conditions of exposure to low temperatures can be perceived as comfortable and without exposure to health risks when evaluated using only the parameters established in ISO standards.

Keywords: Worker's health, Cold, ISO 7730.

\section{INTRODUÇÃO}

A indústria brasileira de abate e processamento de frangos tem apresentado constante crescimento nas últimas décadas, encontrando-se como o terceiro maior produtor mundial de frangos com destaque na exportação, fornecendo carne de 
frango para cerca de 150 países, respondendo por 1,8\% do Produto Interno Bruto (PIB) nacional. A expectativa é que, até 2020, a produção nacional de frango alcance $48,1 \%$ das exportações mundiais (IBGE, 2016; MAPA, 2016).

Esses dados representam o expressivo número de empresas frigoríficas e abatedouros de frangos em atividade e a perspectiva de crescimento desse ramo de atividade no território brasileiro. Em função deste cenário, a necessidade de mão de obra para atender a demanda produtiva torna-se elevada. Segundo a União Brasileira de Avicultura (UBABEF), a avicultura emprega aproximadamente 3,6 milhões de pessoas, direta e indiretamente, envolvendo desde trabalhadores do campo até os trabalhadores das plantas frigoríficas (UBABEF, 2016).

Em contrapartida ao crescimento positivo das empresas na produção de carne de frango, é apontada uma grave problemática em relação às condições de saúde dos trabalhadores que atuam diretamente nas plantas frigoríficas, evidenciada através de estudos nacionais e internacionais que relatam a diversidade de riscos físicos (ruído, temperaturas extremas, umidade, vibração), químicos, biológicos e ergonômicos presentes nas atividades de abate e processamento de frangos e outros animais (ARMSTRONG et al., 1993; BAO; SILVERSTEIN; COHEN, 2001; BONDE et al., 2003; BUSNELLO; DEWES, 2013; CASO; RAVAIOLI; VENERI, 2007; DEJOURS; ABDOUCHELI; JAYET, 1994; FROST; ANDERSEN; NIELSEN, 1998; HECK, 2013; JAKOBI et al., 2015; JUUL-KRISTENSEN et al., 2002; MARRAS, 1993; MUSOLIN et al., 2014; SOMMERICH; MCGLOTHLIN; SUNDSTRUP et al., 2013; SUNDSTRUP et al., 2014).

As atuais estimativas do Sistema de Previdência Social Brasileiro (INSS) reportam que há um elevado índice, cuja média atinge em média $23 \%$ da mão de obra do setor frigorífico, na condição de afastados do trabalho ou no aguardo de decisões judiciais em função de acidentes e doenças ocupacionais (INSS, 2017).

Em destaque a diversidade de condições de risco a saúde e integridade física dos trabalhadores, a exposição a baixas temperaturas ocorre de forma continua e habitual para a maioria dos trabalhadores do ramo de frigorífico e abatedouro e para agravar a exposição ao frio nestas empresas, a maioria das atividades são de forma estática que diminuem a geração de calor do corpo, pois o processo produtivo ocorre de forma sequencial, com maior enfoque na movimentação dos membros 
superiores (REIS, 2012; SARDÁ; RUIZ; KIRTSCHIG, 2009; ILMARINEN; TAMMELA, 1990).

Os setores de após o processo e onde ocorre o corte das partes dos frangos para a industrialização, todos possuem controle de temperatura artificial, o qual obrigatoriamente a maior temperatura do ambiente, segundo os critérios normativos de legislação alimentar no Brasil (MAPA, 1998) determina que a máxima temperatura não deva ultrapassar $15^{\circ} \mathrm{C}$, temperatura considerada fria para os trabalhadores (HOLMÉR, 1997).

A exposição a baixas temperaturas acaba se tornando inevitável em função da necessidade de atendimento da portaria 210 de 10/11/1998 do Ministério da Agricultura e do Abastecimento do Brasil, obrigatória para atividades de cortes de carnes. Esta portaria estabelece limites para diversos setores, em específico na sala de cortes de frangos, onde se concentra a maior quantidade de trabalhadores dos frigoríficos. A portaria determina que a temperatura do frango não deva ser superior à $7^{\circ} \mathrm{C}$ e a temperatura do ambiente não deve ser superior aos $15^{\circ} \mathrm{C}$ (dependendo da região geográfica em que o frigorífico está instalado, sendo 15ㄷ a máxima) (MAPA, 1998). No Brasil, a falta de atendimento a essas temperaturas resulta no impedimento de produzir enquanto as temperaturas forem superiores as estabelecidas na portaria 210. Em virtude da exposição a baixas temperaturas, se não forem controlados adequadamente a exposição dos trabalhadores, o resultado pode ser o aumento significativo de queixas e afastamentos para tratamento de doenças e lesões de acidentes de trabalho (ASSUNÇÃO; ALMEIDA, 2003).

A determinação de medidas que atendam as normativas de conservação do produto é imprescindível para garantir a higiene alimentar e qualidade do produto, todavia, deve haver equilíbrio, para que as variáveis de conforto, segurança e saúde do trabalhador sejam atendidas assim como as normas de higiene alimentar.

Consecutivamente estudos em frigoríficos apontam a prevalência de queixas de dor e desconforto em mais de $80 \%$ dos trabalhadores expostos ao frio do ambiente de trabalho e ao manuseio de produtos congelados. Os trabalhadores se queixam de mãos frias por que precisam manusear pedaços de frangos resfriados e congelados com temperaturas inferiores a $7^{\circ} \mathrm{C}$ (BUZANELO, 2003; BUZANELO; MORO, 2012; HOLMÉR, 1994). 


\section{CONFORTO TÉRMICO}

Os estudos de conforto térmico visam, de maneira geral, analisar e estabelecer condições necessárias para um ambiente térmico adequado às atividades e ocupações humanas, bem como estabelecer métodos e princípios para uma detalhada análise térmica de um ambiente (LAMBERTS; XAVIER, 2012).

A definição clássica de conforto térmico, de acordo com a American Society of Heating, Refrigerating and Air-Conditioning Engineers (ASHRAE) 55, é: "uma condição da mente que expressa satisfação do individuo com o ambiente térmico" (ASHRAE 55, 2013). A esta definição atribui-se que o conforto térmico é uma sensação subjetiva, ou seja, depende das preferências dos indivíduos (LEHMUSKALLIO; HASSI; KETTUNEN, 2002).

Atenção deve se dar as preferências de temperaturas, pois variam muito entre os indivíduos e não há uma temperatura que pode satisfazer a todos (EPSTEIN; MORAN, 2006). Em contrapartida para satisfazer um indivíduo exposto ao frio, ele não pode sentir nenhum tipo de desconforto térmico localizado (FANGER, 1970).

$O$ estudo do conforto térmico e das avaliações das sensações térmicas humanas é representado pelo pesquisador Povl Ole Fanger, onde as equações e métodos desenvolvidos por ele são utilizados como principal referência mundial para elaboração de pesquisas e normas internacionais, como a ASHRAE 55 (2013) e a Organização Internacional de Normalização (ISO) 7730 (2005).

Para identificar a condição de conforto térmico a ISO 7730 (2005), em seu anexo D, considera como aceitável termicamente, conforme procedimento de análise determinado na norma, o ambiente que apresentar um percentual de pessoas insatisfeitas inferior a $10 \%$.

Considerando os métodos das normas ISO e seus anexos para avaliação de exposição ao frio artificial, o objetivo desse estudo foi analisar a exposição a baixas temperaturas em atividades de cortes de frangos e verificar se há exposição a riscos à saúde ocupacional de acordo com os resultados da avaliação determinada pelas normas ISO e dos resultados da avaliação utilizando um sistema de equipamentos para monitoramento de temperatura corporal.

Ressalta-se que a falta de controle da exposição ao frio pode resultar em sintomas localizados como desconforto, dormência, rigidez nas partes com maior 
intensidade de exposição ao frio, redução da destreza, formigamento, redução da sensibilidade dos dedos e flexibilidade das articulações, entre outras anomalias, aumentando consequentemente e predispondo os trabalhadores expostos a essas condições em riscos de acidentes e principalmente problemas de saúde (ARAUJO et al., 2013; HASSI et al., 2001; IIDA, 2005; REIS, 2012).

\section{PROCEDIMENTO METODOLÓGICO}

\subsection{Objeto de Estudo}

Buscou-se com esta pesquisa analisar as condições de exposição ao frio e conforto térmico utilizando dois parâmetros de análise, o critério estabelecido pela norma ISO 7730 (2005) e seus anexos e o sistema de monitoramento de temperatura corporal e ambiental desenvolvido no laboratório de meios porosos e propriedades termofísicas (LMPT) da Universidade Federal de Santa Catariana (GÜTHS et al., 2017).

A pesquisa foi realizada em um frigorífico de abate e processamento de frangos localizado na região de Santa Catarina.

A amostra foi realizada com dados de 32 trabalhadores, no qual todos participantes fazem parte do quadro do setor de cortes, das linhas de produção de peito, perna e asa. Não foram avaliados trabalhadores com menos de um mês de empresa ou em desempenho das atividades.

A jornada de trabalho nas linhas de produção durante o período de coleta de dados iniciava-se às $06 \mathrm{~h}$ : 00min, com a primeira pausa ocorrendo no intervalo entre 07h: $20 \mathrm{~min}$ às $07 \mathrm{~h}: 40 \mathrm{~min}$, segunda pausa entre 09h: $20 \mathrm{~min}$ e 09h: 40min, intervalo de refeição entre $11 \mathrm{~h}: 20 \mathrm{~min}$ às $12 \mathrm{~h}: 20 \mathrm{~min}$, a terceira pausa entre $14 \mathrm{~h}: 00 \mathrm{~min}$ e 14h: $20 \mathrm{~min}$ e término da jornada às $15 \mathrm{~h}$ : $48 \mathrm{~min}$. A jornada é de $8 \mathrm{~h} 48 \mathrm{~min} / \mathrm{dia}$, com intervalo de 60 minutos para alimentação e com pausas de recuperação psicofisiológica de 60 minutos distribuídas na jornada de trabalho em três pausas de 20 minutos, para cumprimento da NR 36 - Segurança e Saúde no Trabalho em Empresas de Abate e Processamento de Carnes e Derivados (NR 36, 2016).

Para realização do estudo foi disponibilizado uma sala com temperatura ambiente média de $23,2^{\circ} \mathrm{C}$, esta temperatura foi medida com um medidor de estres- 
se térmico marca Instrutherm, modelo TGD-300.

Cada trabalhador participante da pesquisa antes de iniciar suas atividades na linha de produção foi direcionado para a sala onde foram instalados os termistores na mão e no pé. Ao chegar à sala o procedimento realizado pelos pesquisadores consistia em retirar a botina e a meia do pé do trabalhador, instar os termistores, recolocar a meia e botina e em seguida instalar os termistores na mão e posteriormente recolocar as luvas e mangotes. O tempo médio de instalação de todos os termistores foi de aproximadamente 5 minutos por trabalhador, instalados por dois pesquisadores. Durante toda a jornada, intervalo e pausas o trabalhador permaneceu com os termistores instalados e somente no final da jornada retornava para a sala para retirada dos termistores e para o preenchimento dos dados do perfil do trabalhador.

Conforme análise de risco descrita no programa de prevenção de riscos ambientais (PPRA) da empresa, em todas as atividades avaliadas nessa pesquisa é obrigatória a utilização na mão que segura o produto: luva de algodão sobreposta com luva nitrílica sobreposta com luva malha de aço e na mão que segura à faca, luva de algodão sobreposta com luva nitrílica. Nos pés, meia de algodão e botina de Policloreto de Vinila (PVC), todos os equipamentos são fornecidos pela empresa. A meia possui certificação para exposição ao frio e a botina possui certificação para exposição à umidade. Durante a pesquisa foram entregues meias e luvas para os trabalhadores que por algum motivo específico não possuíam a luva ou meia conforme determinação do PPRA. Todos foram entregues apenas no dia da avaliação no trabalhador e para os que utilizam meias e luvas adicionais foram solicitados que não utilizassem no dia da avaliação.

\subsection{Procedimentos de Coleta de Dados}

Para avaliar a condição de conforto pela norma ISO 7730 (2005) em locais com baixas temperaturas, utilizam-se as variáveis pessoais e ambientais. As variáveis pessoais mensuradas foram: taxa metabólica e isolamento das roupas. As variáveis ambientais: temperatura do ar, velocidade relativa do ar, temperatura média radiante e umidade relativa do ar. 
Para classificação da taxa metabólica das atividades avaliadas foi utilizado os anexos da ISO 8996 (2004). Considerando que no setor pesquisado todos os participantes do estudo realizam a mesma tarefa que é cortar peças de frango, a qual consiste em pegar e manipular os pedaços de frango com uma das mãos, de forma que facilite o direcionamento do corte com a faca sustentada com a mão contrária. Nessa atividade durante todo o tempo de trabalho a posição dos indivíduos é em ortostase e estática. Com essas características da atividade, foi classificada de acordo com a ISO 8996 (2004) como atividade de baixa taxa metabólica.

Os dados que representam as condições do setor e as temperaturas das mãos e pés dos trabalhadores foram coletados durante toda a jornada de trabalho diária por um período de 17 dias de avaliação.

O sistema de monitoramento de temperatura corporal utilizado nesse estudo consiste em uma rede de sensores sem fio (WSN) usando a tecnologia Zigbee, no qual um conjunto de termistores se destina a medir a temperatura corporal dos trabalhadores, enquanto o outro conjunto avalia as variáveis ambientais.

Ambos os módulos de sensores enviam os dados amostrados em tempo real através de um link de rádio através de um conversor USB-serial conectado a um computador.

No estudo foram utilizados dois módulos de sensores em cada trabalhador avaliado (item 2 - Quadro 1), o qual um módulo foi utilizado para medir a temperatura no dedo indicador, centro da mão e punho e o outro módulo foi utilizado para medir a temperatura do hálux e região central na parte superior do pé. Em cada dia de avaliação foram monitorados dois trabalhadores durante toda a jornada diária.

Os termistores foram instalados em todos trabalhadores na mão que segura 0 produto e no pé correspondente ao mesmo lado (direito ou esquerdo) dos termistores da mão.

Os pontos corporais avaliados na mão e pé foram selecionados fundamentados na ISO 11079 (2007) "Ergonomics of the termal environment" a qual não determina pontos específicos a serem avaliados para exposições ao frio localizado, mas recomenda controle frequente de temperatura dos dedos e sugere que as temperaturas devem estar acima de $24^{\circ} \mathrm{C}$ para a preservação e bom funcionamento das mãos. Também determina que a quantidade de conhecimento 
sobre as respostas a resfriamento local é insuficiente para o desenvolvimento de um método de avaliação único e que pesquisas sobre o assunto devem ser incentivadas (ISO 11079, 2007).

Os termistores foram fixados com fita microporosa diretamente na pele do trabalhador (item 1 - Quadro 1).

Após a instalação dos sensores, os trabalhadores foram vestidos pelos pesquisadores dos demais equipamentos de proteção obrigatórios para executar suas atividades diárias, a ilustração de um trabalhador com os módulos de sensores e todos os equipamentos para executar suas atividades é representado no item 2 Quadro 1.

O módulo de medição das variáveis ambientais foi montado próximo às estações de trabalho onde foram coletados os dados de temperatura corporal, conforme ilustrado na figura apresentada no item 3- Quadro 1.

As variáveis ambientais coletadas pelo módulo de medição ambiental determinam dados que subsidiam a avaliação de PMV (Voto Médio Estimado) e PPD (Voto Médio Predito). Esses índices são definidos pela ISO 7730 (2005) e ASHRAE 55 (2013), com base em estudos de Fanger (1970), para avaliação de conforto médio de uma pessoa ou um grupo de pessoas que praticam uma atividade.

Para mensuração da temperatura superficial do produto manipulado foi utilizado um termômetro digital infravermelho marca Minipa, modelo MT-350. 
Quadro 1 - Demonstração dos equipamentos de monitoramento e temperatura instalados nos trabalhadores e nos postos de trabalho

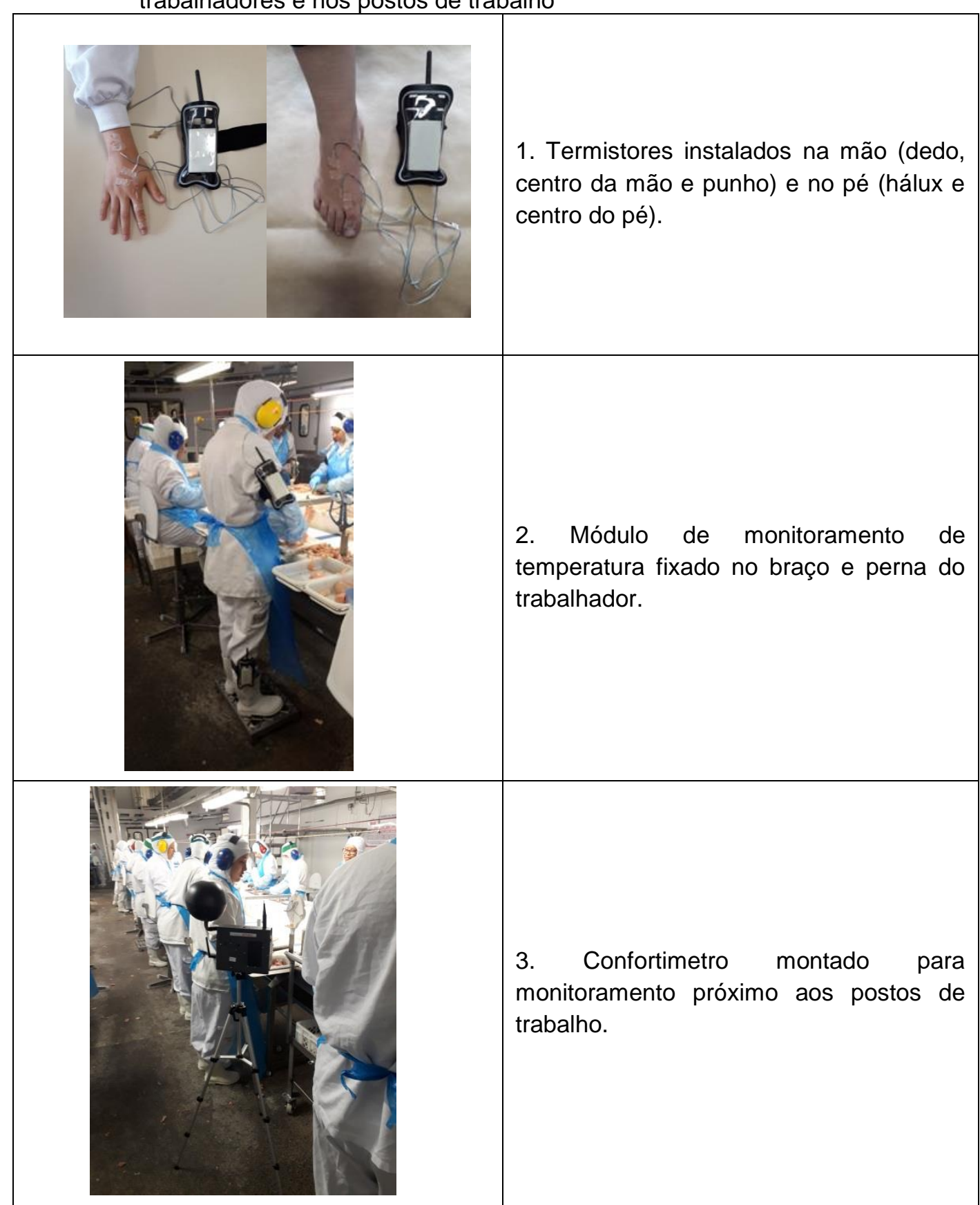

Fonte: Resultados da pesquisa (2018)

\subsection{Procedimentos de Análise de Dados}

Para compilação e análise dos dados utilizou-se da estatística descritiva com cálculo de média, desvio padrão e exclusão dos outliers (critério $|Z|>3=$ Valor outliers). 
Ressalta-se que nesse estudo não foi realizada análise separada dos dados outliers para investigar os casos extremos.

Para comparação das médias dos resultados foi utilizado o resultado conforme atendimento do calculado do teste $F$ na análise de variância (ANOVA) com nível de significância de 5\%.

Foram utilizados os valores médios calculados dos resultados das avaliações no início da atividade, saída e retorno de cada pausa psicofisiológica, saída e retorno do intervalo de refeição e término da jornada de trabalho.

Como parâmetros para análise dos dados foi utilizado uma adaptação das escalas de temperatura, baseada no estudo conduzido por Lehmuskallio, Hassi e Kettunen (2002), conforme Tabela 1.

Tabela 1 - Escala adaptada de possível sensação térmica

\begin{tabular}{lll}
\hline Escala (ㅜ) & \multicolumn{1}{c}{ Possível sensação do indivíduo } & \multicolumn{1}{c}{ Adaptação } \\
\hline $36-34$ & Indica condição de conforto térmico & Conforto \\
$33,9-29$ & Indica condição neutra & Neutro \\
$28,9-25$ & Indica desconforto (resfriado) & Resfriado \\
$24,9-18$ & Indica sensação de desconforto (frio) & Frio \\
$17,9-12$ & Indica sensação de dor provocada pelo frio & Dor \\
$11,9-8$ & Indica sensação de dor e formigamento (perda gradual da & Dor e \\
Menor de 8 & Indica sensação de dor e congelamento & formigamento \\
\hline
\end{tabular}

Fonte: Adpatado de Lehmuskallio, Hassi e Kettunen (2002)

Para análise dos dados do voto médio predito (PMV) foi utilizado a escala de 7 pontos da ISO 7730 (2005), onde: (-3) muito frio; (-2) frio; (-1) levemente frio; (0) neutro; $(+1)$ levemente quente; $(+2)$ quente; $(+3)$ muito quente.

Essa pesquisa foi autorizada pelo representante legal da empresa que concedeu o local para realizar as avaliações e todas as funcionárias participantes aceitaram o termo de consentimento livre esclarecido conforme protocolo da pesquisa, submetida e aprovada pela Comissão de Ética em Pesquisa da Universidade Federal de Santa Catarina, atendendo a todas as recomendações legais. $O$ anonimato e confidencialidade das informações foram mantidos nos registros dos dados. 


\section{RESULTADOS}

\subsection{Resultados de acordo com as normas ISO - variáveis pessoais}

Seguindo os procedimentos estabelecidos, inicialmente foi classificada a taxa de metabolismo como baixa. A atividade foi comparada na tabela de gasto metabólico da ISO 8996 (2004) como atividade utilizando as mãos e braços com ferramenta de pequeno porte com manuseio de materiais leves. Para essa classificação da atividade a taxa de metabolismo foi atribuída $130 \mathrm{~W} / \mathrm{m}^{2}$ equivalente a 2,2 met.

O segundo item avaliado foi o índice de resistência térmica das vestimentas dos trabalhadores. A análise foi realizada conforme resultado do somatório final de todas as vestimentas de cada indivíduo considerando os índices de isolamento térmico para vestimentas da ISO 9920 (2007), que determina o índice de isolamento térmico (ICl) para as roupas.

Os resultados apontam variações no isolamento térmico total (Tabela 3) devido às vestimentas adicionais aos uniformes utilizadas por cada trabalhador, por exemplo, o uso de blusas e calças por baixo do uniforme, conforme as preferências individuais.

O uniforme e equipamentos entregues pela empresa para os trabalhadores da linha de produção em estudo são: touca (poliéster), blusa (65\% poliéster e $35 \%$ algodão), calça (65\% poliéster e $35 \%$ algodão), meia grossa (algodão), bota cano alto de PVC, luva de lã, luva nitrílica, luva de aço e avental fino descartável (plástico). Ressalta-se que esse uniforme é entregue para todos os trabalhadores que exercem atividades em locais com temperaturas superiores a $10^{\circ} \mathrm{C}$, indiferente do setor na empresa em estudo.

Analisando a ISO 9920 (2007) o Icl do conjunto de uniformes e equipamentos disponibilizados pela empresa é de $0,87 \mathrm{clo}$, portanto considerando que todos participantes do estudo é do sexo feminino, e que conforme dados coletados, para completar a vestimenta de trabalho somou-se ao conjunto entregue pela empresa as roupas: calcinha + sutiã, totalizando 0,9 clo a vestimenta fornecida para executar o trabalho padrão. 


\subsection{Resultados de acordo com os critérios das normas ISO - variáveis ambientais}

Com relação às variáveis ambientais, na Tabela 2 estão representadas as médias calculadas em cada momento da jornada que inicia e finaliza um ciclo de trabalho.

Tabela 2 - Resultados das avaliações ambientais nos postos de trabalho

\begin{tabular}{lcccccc}
\hline \multicolumn{2}{c}{ Jornada de trabalho } & \multicolumn{5}{c}{ Variáveis ambientais } \\
Início & TBS $\left({ }^{\circ} \mathbf{C}\right)$ & TG $\left({ }^{\circ} \mathbf{C}\right)$ & TP $\left({ }^{\circ} \mathbf{C}\right)$ & Var $(\mathbf{m} / \mathbf{s})$ & UR (\%) \\
\hline Saída pausa & 06h:00mim & 13,5 & 13,4 & 3,9 & 0,36 & 83 \\
Retorno pausa & 07h:20mim & 12,3 & 11,9 & 3,6 & 0,34 & 82 \\
Saída pausa & 09h:40mim $: 20 \mathrm{~mm}$ & 11,5 & 11,4 & 3,7 & 0,34 & 83 \\
Retorno pausa & $09 \mathrm{~h}: 40 \mathrm{mim}$ & 11,4 & 11,3 & 4,0 & 0,34 & 85 \\
Saída intervalo & $11 \mathrm{~h}: 20 \mathrm{mim}$ & 12,2 & 12,5 & 3,9 & 0,36 & 84 \\
Retorno intervalo & $12 \mathrm{~h}: 20 \mathrm{mim}$ & 12,9 & 12,6 & 4,1 & 0,35 & 82 \\
Saída pausa & $14 \mathrm{~h}: 00 \mathrm{mim}$ & 11,4 & 11,2 & 4,2 & 0,33 & 82 \\
Retorno pausa & $14 \mathrm{~h}: 20 \mathrm{mim}$ & 11,4 & 11,3 & 4,0 & 0,33 & 84 \\
Término & 15h:45mim & 12,4 & 12,5 & 4,4 & 0,35 & 82 \\
& Média & $\mathbf{1 2 , 0}$ & $\mathbf{1 1 , 9}$ & $\mathbf{4 , 0}$ & $\mathbf{0 , 3 4}$ & $\mathbf{8 3}$ \\
\hline
\end{tabular}

Nomenclatura: TBS - termômetro de bulbo seco; TG - termômetro de globo; TP - temperatura do produto; Var - Velocidade do ar; UR - Umidade.

Fonte: Resultados da pesquisa (2018)

O resultado médio final para tbs foi de $12^{\circ} \mathrm{C}$, a tg foi de $11,9^{\circ} \mathrm{C}$, a var foi de $0,34 \mathrm{~m} / \mathrm{s}$ (sistema de ventiladores da sala ligados) e a umidade foi de $83 \%$. A temperatura média da peça de frango manipulado pelos trabalhadores foi de $4,0^{\circ} \mathrm{C}$.

Conhecendo-se os parâmetros pessoais, a resistência térmica das vestimentas, a taxa metabólica e as variáveis ambientais, de acordo com a ISO 7726 (1998), calculou-se o Voto Médio Estimado (PMV) e o Voto Médio Predito (PPD), os resultados são apresentados na Tabela 03. 
Tabela 3 - Resultados das variáveis pessoais e ambientais conforme critérios da ISO 7730/05

\begin{tabular}{|c|c|c|c|c|c|c|c|}
\hline \multirow{2}{*}{ Participantes } & \multirow{2}{*}{$\begin{array}{l}\text { Uniforme } \\
\text { Icl (clo) }\end{array}$} & \multicolumn{2}{|c|}{$\begin{array}{c}\text { Uniforme + } \\
\text { vestimentas }\end{array}$} & \multicolumn{2}{|c|}{ Uniforme } & \multicolumn{2}{|c|}{$\begin{array}{c}\text { Uniforme + } \\
\text { vestimentas }\end{array}$} \\
\hline & & $\begin{array}{l}\text { IcI R } \\
\text { (clo) }\end{array}$ & $\begin{array}{l}\text { Icl T } \\
\text { (clo) }\end{array}$ & PMV & PPD (\%) & PMV & PPD (\%) \\
\hline 1 & 0,9 & 0,7 & 1,6 & $-0,23$ & 6,13 & 0,53 & 12,86 \\
\hline 2 & 0,9 & 0,8 & 1,7 & $-0,23$ & 6,13 & 0,6 & 15,84 \\
\hline 3 & 0,9 & 0,9 & 1,8 & $-0,23$ & 6,13 & 0,66 & 19,17 \\
\hline 4 & 0,9 & 0,7 & 1,6 & $-0,23$ & 6,13 & 0,53 & 12,86 \\
\hline 5 & 0,9 & 0,6 & 1,5 & $-0,23$ & 6,13 & 0,45 & 10,32 \\
\hline 6 & 0,9 & 0,9 & 1,8 & $-0,23$ & 6,13 & 0,66 & 19,17 \\
\hline 7 & 0,9 & 0,7 & 1,6 & $-0,23$ & 6,13 & 0,53 & 12,86 \\
\hline 8 & 0,9 & 0,7 & 1,6 & $-0,23$ & 6,13 & 0,53 & 12,86 \\
\hline 9 & 0,9 & 0,5 & 1,4 & $-0,23$ & 6,13 & 0,36 & 8,24 \\
\hline 10 & 0,9 & 0,9 & 1,8 & $-0,23$ & 6,13 & 0,66 & 19,17 \\
\hline 11 & 0,9 & 0,7 & 1,6 & $-0,23$ & 6,13 & 0,53 & 12,86 \\
\hline 13 & 0,9 & 0,6 & 1,5 & $-0,23$ & 6,13 & 0,45 & 10,32 \\
\hline 14 & 0,9 & 0,8 & 1,7 & $-0,23$ & 6,13 & 0,6 & 15,84 \\
\hline 15 & 0,9 & 0,3 & 1,2 & $-0,23$ & 6,13 & 0,17 & 5,59 \\
\hline 16 & 0,9 & 0,6 & 1,5 & $-0,23$ & 6,13 & 0,45 & 10,32 \\
\hline 17 & 0,9 & 0,7 & 1,6 & $-0,23$ & 6,13 & 0,53 & 12,86 \\
\hline 18 & 0,9 & 0,7 & 1,6 & $-0,23$ & 6,13 & 0,53 & 12,86 \\
\hline 20 & 0,9 & 0,6 & 1,5 & $-0,23$ & 6,13 & 0,45 & 10,32 \\
\hline 21 & 0,9 & 0,8 & 1,7 & $-0,23$ & 6,13 & 0,6 & 15,84 \\
\hline 22 & 0,9 & 0,8 & 1,7 & $-0,23$ & 6,13 & 0,6 & 15,84 \\
\hline 23 & 0,9 & 0,7 & 1,6 & $-0,23$ & 6,13 & 0,53 & 12,86 \\
\hline 24 & 0,9 & 0,9 & 1,8 & $-0,23$ & 6,13 & 0,66 & 19,17 \\
\hline 25 & 0,9 & 0,6 & 1,5 & $-0,23$ & 6,13 & 0,45 & 10,32 \\
\hline 26 & 0,9 & 0,8 & 1,7 & $-0,23$ & 6,13 & 0,6 & 15,84 \\
\hline 27 & 0,9 & 0,5 & 1,4 & $-0,23$ & 6,13 & 0,36 & 8,24 \\
\hline 28 & 0,9 & 0,7 & 1,6 & $-0,23$ & 6,13 & 0,53 & 12,86 \\
\hline 29 & 0,9 & 0,8 & 1,7 & $-0,23$ & 6,13 & 0,6 & 15,84 \\
\hline 30 & 0,9 & 0,6 & 1,5 & $-0,23$ & 6,13 & 0,45 & 10,32 \\
\hline 31 & 0,9 & 0,7 & 1,6 & $-0,23$ & 6,13 & 0,53 & 12,86 \\
\hline 32 & 0,9 & 0,8 & 1,7 & $-0,23$ & 6,13 & 0,6 & 15,84 \\
\hline Média & 0,9 & 0,7 & 1,6 & $-0,23$ & 6,13 & 0,53 & 12,86 \\
\hline
\end{tabular}

Nomenclaturas: Icl - Índice de isolamento térmico; Icl $\mathrm{R}$ - Índice de isolamento térmico das vestimentas adicionais de cada trabalhador; Icl T - Somatório total do índice de isolamento térmico, clo - Unidade de medição da resistência térmica da roupa; PMV - Voto médio predito; PPD - Porcentagem de pessoas insatisfeitas.

Fonte: Resultados da pesquisa (2018)

Os resultados da Tabela 3 demonstram que considerando que a atividade, os uniformes e equipamentos são similares para os 32 participantes do estudo, os resultados de PMV e PPD\% (Uniforme) calculados são idênticos nessas condições. Os resultados variam quando se considera as vestimentas individuais utilizadas 
conforme utilização de roupas adicionais devido às preferências de cada participante do estudo.

Comparando os resultados da Tabela 3 com a escala de avaliação da ISO 7730 (2005): muito frio (-3); frio (-2); levemente frio (-1); neutro (0); levemente quente $(+1)$; quente $(+2)$; e muito quente $(+3)$, verifica-se que, considerando o conjunto de uniformes e equipamentos cedidos pela empresa às avaliações de PMV provê uma sensação que varia entre neutro e levemente frio.

Ao comparar os resultados do conjunto de uniformes e equipamentos + vestimentas utilizadas por cada participante do estudo o PMV provê uma sensação que varia entre neutro e levemente quente.

Os resultados também mostram a percentagem de pessoas que estariam insatisfeitas com as condições térmicas do ambiente. Trata-se do PPD que no estudo representou o valor de $6,1 \%$ considerando apenas o conjunto de uniforme e equipamentos cedidos pela empresa. Em contrapartida os resultados considerando o conjunto de uniforme e equipamentos + as vestimentas individuais demonstram resultados de insatisfação com um PPD médio de 12,8\%. Esse resultado pode ser atribuído a diversas situações, que podem ser desde a necessidade individual de proteção ao frio, à falta de conhecimento técnico de regulação de proteção necessária para uso de vestimentas adicionais, condições climáticas externas.

\subsection{Resultados das avaliações utilizando o sistema de avaliação de temperatura corporal}

Com relação às temperaturas avaliadas nos sítios corporais da mão e do pé de cada participante, na Tabela 4 estão representadas as médias calculadas em cada momento da jornada que inicia e finaliza um ciclo de trabalho.

Verifica-se nos resultados que ocorrem variações de temperatura em todos os sítios corporais avaliados, porem as menores temperaturas mensuradas foram registradas no horário de saída para o intervalo de refeição, às 11h: 20min. 
Tabela 4 - Escala adaptada de possível sensação térmica

\begin{tabular}{lcccccc}
\hline \multirow{2}{*}{ Jornada de trabalho } & \multicolumn{3}{c}{ Mão } & \multicolumn{2}{c}{ Pé } \\
& & Dedo & Centro & Punho & Hálux & Centro \\
\hline Início & 06h: 00mim & 30,0 & 33,4 & 34,0 & 29,2 & 33,0 \\
Saída pausa & 07h: 20mim & 18,8 & 27,6 & 29,5 & 19,0 & 29,7 \\
Retorno pausa & 07h: 40mim & 20,3 & 28,5 & 30,0 & 18,9 & 30,0 \\
Saída pausa & 09h: 20mim & 14,0 & 24,9 & 29,4 & 14,2 & 26,1 \\
Retorno pausa & 09h: 40mim & 16,2 & 27,1 & 30,0 & 15,3 & 26,9 \\
Saída intervalo & 11h: 20mim & 14,2 & 24,6 & 29,7 & 13,6 & 23,3 \\
Retorno intervalo & 12h: 20mim & 24,1 & 31,0 & 32,6 & 21,7 & 30,0 \\
Saída pausa & 14h: 00mim & 16,8 & 27,3 & 30,2 & 16,0 & 27,6 \\
Retorno pausa & 14h: 20mim & 17,9 & 28,8 & 31,9 & 15,4 & 27,5 \\
Término & 15h: 45mim & 16,3 & 26,1 & 30,3 & 14,8 & 25,0 \\
\hline \multicolumn{7}{l}{ Fonte: Resultados da pesquisa (2018) }
\end{tabular}

Percebe-se que de acordo com os resultados, de todos os sítios corporais, a média de temperatura no início da jornada representa as maiores temperaturas em cada sitio corporal avaliado. Porem ao avaliar a Tabela 2 percebe-se que o ambiente de trabalho já está com temperaturas baixas, bem próximas às temperaturas mensuradas durante toda a jornada de trabalho. Provavelmente isso ocorre por que as temperaturas externas em que o trabalhador esta exposto apresentam temperaturas acima das encontradas no ambiente de trabalho, pois se ressalta que a pesquisa ocorreu na estação climática do período de verão.

Também se verifica nos resultados que as menores temperaturas foram registradas no dedo da mão e no hálux.

\section{DISCUSSÕES}

Inicialmente verifica-se que de acordo com o índice de isolamento do conjunto de uniforme e equipamentos cedidos pela empresa, o valor de resistência térmica calculado seguindo os parâmetros da ISO não atende as necessidades de nenhum dos participantes do estudo. Percebeu-se uma variedade elevada de uso de vestimentas para exposição ao frio (blusas e calças) por razões que podem ser confirmadas nos estudos realizados por Nicol (1993), Xavier (2000) e Nicol e Humphreys (2002) que descrevem que o modelo de Fanger utilizado na ISO 7730 (2005), prove vestimentas de forma geral onde não leva em conta a adaptação, os 
hábitos e estilos de vida das pessoas, aspectos de natureza física, psicológica e fisiológica.

Também se ressalta que o índice de isolamento foi considerado para vestimentas em perfeitas condições de uso, pois quando comparadas subjetivamente com a tabela da ISO 9920 (2007) não há dados que consideram o desgaste decorrente do uso das vestimentas, fator que também pode contribuir para a sensação de frio que contribui diretamente para o uso elevado número de vestimentas utilizadas por cada participante do estudo para complementar o isolamento térmico necessário.

O resultado da necessidade de isolamento térmico também pode ser confirmado nos resultados do PMV do conjunto de uniformes e equipamentos cedidos pela empresa, o qual apresentou resultado de -0,23 que é considerado como uma condição de sensação térmica caracterizada como neutra (0) para leve frio $(-1)$.

Ao avaliar os resultados do PMV considerando o somatório do índice de isolamento térmico do uniforme e vestimentas adicionais utilizadas conforme a necessidade de cada trabalhador, a sensação média de todos os resultados é $+0,53$, a qual é interpretada como condição entre neutro (0) e levemente quente $(+1)$. Condição que de acordo com os parâmetros da escala de sensação térmica da ISO 7730 (2005) não são adequadas, pois a condição aceitável é $-0,5<P M V<+0,5$.

O resultado do PPD, considerando apenas os conjuntos de uniformes e equipamentos cedidos pela empresa, apresenta que atendem o índice com um valor $<10 \%$ e o resultado do somatório das vestimentas cedidas pela empresa + vestimentas individuais apresentou resultado pouco acima de $10 \%$ da porcentagem de insatisfeitos, o que leva a concluir que conforme os parâmetros da ISO 7730 (2005) os uniformes e equipamentos cedidos pela empresa atentem a necessidade de acordo com atividade e a auto regulação apresentada com vestimentas adicionadas individualmente não é satisfatória para a maioria dos indivíduos do estudo.

Convém destacar que experiências realizadas por Fanger (1970), Rohles (1971) e Nevins et al. (1966) provaram que é impossível obter em um ambiente combinação das variáveis de conforto que satisfaça plenamente todos os integrantes de um grupo; sempre existirão insatisfeitos pois envolvem questões subjetivas e 
individuais. No estudo essa condição pode ser confirmada pela diferença de vestimentas utilizadas por baixo dos uniformes, que variam em sua resistência térmica de 0,3 clo a 0,9 clo.

O que se percebe nos resultados desse estudo é que as vestimentas cedidas apresentam sensação de leve condição de frio e que nessas condições de acordo com a ISO 7730 (2005) a sensação é considerada para maioria como satisfatória. Porém não é o que ocorre, pois conforme resultados das vestimentas individuais, todos participantes demonstraram necessidade de complementar as vestimentas cedidas pela empresa com o uso de roupas para temperaturas baixas, como blusas e calças. Não foram encontrados nos resultados participantes utilizando apenas roupas intimas e camisetas por baixo do uniforme e equipamentos, condição que é encontrada em outros setores do mesmo abatedouro com exposição a temperaturas ambientais próximas de $25^{\circ} \mathrm{C}$.

Em contrapartida os resultados obtidos com o sistema de monitoramento de temperatura corporal, demonstram riscos à saúde dos trabalhadores quando comparados aos parâmetros estabelecidos no estudo de Lehmuskallio, Hassi e Kettunen (2002). Os resultados são apresentados na Tabela 5.

Tabela 5 - Resultados das subjetivas sensações conforme parâmetros de Lehmuskallio, Hassi e Kettunen (2002)

\begin{tabular}{lc|c|c|c|c|c}
\hline \multirow{2}{*}{ Jornada de trabalho } & \multicolumn{4}{c|}{ Mão } & \multicolumn{2}{c}{ Pé } \\
\cline { 3 - 7 } & & Dedo & Centro & Punho & Hálux & Centro \\
\hline Início & 06h: $00 \mathrm{mim}$ & Neutro & Neutro & Conforto & Neutro & Neutro \\
Saída pausa & 07h: $20 \mathrm{mim}$ & Frio & Resfriado & Neutro & Frio & Neutro \\
Retorno pausa & 07h: $40 \mathrm{mim}$ & Frio & Resfriado & Neutro & Frio & Neutro \\
Saída pausa & 09h: $20 \mathrm{mim}$ & Dor & Frio & Neutro & Dor & Resfriado \\
Retorno pausa & 09h: $40 \mathrm{mim}$ & Dor & Resfriado & Neutro & Dor & Resfriado \\
Saída intervalo & $11 \mathrm{~h}: 20 \mathrm{mim}$ & Dor & Frio & Neutro & Dor & Frio \\
Retorno intervalo & $12 \mathrm{~h}: 20 \mathrm{mim}$ & Frio & Neutro & Neutro & Frio & Neutro \\
Saída pausa & $14 \mathrm{~h}: 00 \mathrm{mim}$ & Dor & Resfriado & Neutro & Dor & Resfriado \\
Retorno pausa & $14 \mathrm{~h}: 20 \mathrm{mim}$ & Dor & Resfriado & Neutro & Dor & Resfriado \\
Término & $15 \mathrm{~h}: 45 \mathrm{mim}$ & Dor & Resfriado & Neutro & Dor & Resfriado \\
\hline
\end{tabular}

Fonte: Resultados da pesquisa (2018)

Percebem-se nos resultados que ao iniciar a atividade a sensação é dedos e centro da mão em condição considerada neutra em relação ao frio, punho com sensação de conforto. No pé a sensação no centro é neutra e no hálux é sensação 
de desconforto causado pelo resfriado. Porem essas condições mantem-se por pouco tempo e conforme dados analisados nesse estudo, após 01h: 20min de trabalho a sensação no dedo da mão e no hálux altera-se para sensação de frio (desconforto). Nota-se que essas condições pioram, passando para sensação de dor conforme o tempo de exposição aumenta.

Os resultados também demonstram que as piores sensações ocorrem no dedo da mão e no hálux com exposição a temperaturas possíveis de causar sensação de dor provocada pelo frio.

Não foram evidenciadas temperaturas que indicam sensação de dor e formigamento e sensação de congelamento.

Os dados que apresentam o resultado mais crítico são na saída para o intervalo, com sensação de dor no dedo da mão, frio no centro da mão, dor no hálux e sensação de frio no centro do pé.

A condição de sensação de dor nos dedos das mãos e pés e a sensação de desconforto nas regiões das mãos e pés mensurados nesse estudo no modelo de atividade avaliada podem ser facilmente confundidas com outras variáveis da atividade. Nos pés em função da postura em ortostase e estática durante todo o tempo de trabalho e nas mãos devido ao ritmo acelerado de movimentos de corte realizados por peça de frango ao dia. Ao mesmo tempo em que esses fatores podem confundir a percepção do individuo exposto aos riscos da atividade avaliada, todas essas condições contribuem no desenvolvimento de queixas de dor e possíveis lesões nas mãos e pés.

Os resultados mensurados por meio do sistema de monitoramento de temperatura corporal detectam temperaturas associadas a sensações que demonstram que é arriscado afirmar que as condições avaliadas seguindo os parâmetros das normas ISO utilizadas nesse estudo propiciam condições de conforto e controle da saúde na exposição às variáveis ambientais e individuais mensuradas nesse modelo de atividade.

Segundo Nicol e Parsons (2002) a norma ISO 7730 (2005) foi baseada em pesquisas realizadas na década de 1960 e posteriormente foram desenvolvidos diversos estudos, onde há demonstrações de discordância com o modelo de avaliação da norma ISO 7730 (2005) sendo elas: não há balanço entre o calor produzido e o calor perdido pelo corpo que é condição necessária para obtenção de 
conforto térmico; o modelo mensura o estado térmico estável; não consideram a adaptação, os hábitos e estilos de vida das pessoas, aspectos de natureza física, psicológica e fisiológica; as tabelas levam a erros, mais de 50 na taxa metabólica e até $25 \%$ no isolamento das roupas; modelo de PMV pode produzir predição errônea sempre que aplicado a um grande grupo de pessoas; limites das temperaturas do ar, a velocidade do ar e as variáveis climáticas instantâneas não refletem todo o período da pesquisa; em câmaras climatizadas não é possível identificar corretamente qual é o fator de insatisfação das pessoas.

Enfim Nicol e Parsons (2002) ressalta que é oportuno realizar uma revisão completa da norma a fim de integrar novos conhecimentos, visto que durante esses anos vários pesquisadores investigaram os aspectos específicos de conforto térmico em laboratório e em campo e encontraram discordâncias diversas no modelo de avaliação de conforto térmico proposto na ISO 7730 (2005), como pode ser observado nos resultados desse estudo.

Pode-se concluir com os dados mensurados nesse estudo com o uso do sistema de monitoramento de temperatura corporal, que o método proposto na ISO 7730 (2005) e suas complementares, assim como o modelo de atividade avaliada necessitam de melhorias que possam propor condições que conservem os indivíduos expostos ao frio em condições de conforto e controle de riscos a saúde. $\mathrm{O}$ sistema de monitoramento de temperatura corporal demonstrou nesse estudo ser um método eficiente na complementação de informações para satisfação do conforto e controle de riscos à saúde causados por trabalho em ambientes com atividades com exposição a baixas temperaturas.

Ressalta-se que como foram avaliados apenas sítios corporais da mão e pé com o sistema de monitoramento de temperatura e o modelo proposto na ISO 7730 (2005) considera a exposição de todo o indivíduo, ou seja, é possível que em certas regiões do corpo como abdome, tórax, pernas e braços, os indivíduos estejam realmente satisfeitos e que não há riscos para saúde nas regiões mais protegidas da exposição a baixas temperaturas.

\section{CONCLUSÕES}

A avaliação subjetiva de parâmetros de segurança e saúde do trabalho para 
exposição ocupacional ao frio dificulta as ações a serem implantadas pelas empresas e confunde o entendimento dos profissionais de medicina e segurança do trabalho para manter condições propícias de trabalho em ambientes com exposição a baixas temperaturas e por sua vez quem é prejudicado diretamente é o individuo exposto a condições inseguras com parâmetros que supostamente são coerentes e as empresas que perdem em produtividade, qualidade e segurança devido à falta de conforto e segurança dos trabalhadores.

Nesse estudo as avaliações realizadas seguindo os parâmetros das normas ISO para avaliação de conforto térmico demonstram que as vestimentas e equipamentos cedidos pela empresa podem ser considerados termicamente como confortáveis e adequados a proteção de riscos à saúde. Porém os resultados do estudo demonstram que existem necessidades individuais de complementar a proteção à exposição a baixas temperaturas por que o índice de resistência térmica das vestimentas e equipamentos não apresenta suficiente para garantir o conforto e consecutivamente o controle de riscos a saúde.

Para confirmar a necessidade de auto regulação devido à percepção de frio nesse modelo de atividade avaliado os resultados das temperaturas mensuradas nos sítios corporais da mão e pé demonstram que realmente as condições são de desconforto, dor e de exposição a riscos à saúde dos indivíduos expostos a baixas temperaturas que não são identificadas e mensuradas no modelo de avaliação proposto pela ISO. Com isso, o resultado desse estudo confirma que é arriscado controlar as condições de exposição ao frio utilizando os critérios propostos nas normas ISO para avaliação de conforto térmico.

Para completar a avaliação do modelo proposto nas normas ISO o sistema de monitoramento de temperatura demonstrou a possibilidade de quantificar as temperaturas em condições reais de trabalho, e não de forma qualitativa e subjetiva com possibilidades de resultados duvidosos, permitindo resultados quantitativos que possibilitam suporte para ações de controle da exposição a baixas temperaturas.

\section{REFERÊNCIAS}

ARAUJO AFDV et al. Identificação de fatores de riscos ocupacionais no processo de abate de bovinos. Cadernos de Pesquisa, v. 19, n. 3, p. 79-89, 2013. 
ARMSTRONG, J.; et al. A conceptual model for workrelated neck and upper-limb musculoskeletal disorders. Scand J Work Environ Health, v. 19, n. 2, p. 73-84, 1993. tps://doi.org/10.5271/sjweh.1494

ASSUNÇÃO, A.; ALMEIDA, I.M. Doenças osteomusculares relacionadas com o trabalho: membro superior e pescoço. 2. ed. São Paulo: Atheneu, 2003.

ASHRAE 55 - Thermal environmental conditions for human occupancy. American Society of Heating, Refrigerating and Air Conditioning Engineers Inc., 2013.

BAO, S.; SILVERSTEIN, B.; COHEN, M. An electromyography study in three high risk poultry processing jobs. International Journal Of Industrial Ergonomics, v. 27, n. 6 , p.375-385, Jun. 2001. Disponível em:

http://api.elsevier.com/content/article/PII:S016981410100004X?httpAccept=text/xml.

Acesso em: 02 out. 2017. https://doi.org/10.1016/S0169-8141(01)00004-X

BONDE, J.P.; et al. Prognosis of shoulder tendonitis in repetitive work: a follow up study in a cohort of Danish industrial and service workers. Occup Environ Med, v. 60, E8, 2003. https://doi.org/10.1136/oem.60.9.e8

BUSNELLO, G.F.; DEWES, M. Doenças Osteomusculares Relacionadas à Atividades de Trabalhadores de Frigoríficos de Frangos. Brazilian Journal Of Surgery And Clinical Research - Bjscr, Maringá, v. 4, n. 3, p. 27-32, 9 set. 2013. Disponível em: http://www.mastereditora.com.br/biscr. Acesso em: 20 out. 2017. https://doi.org/10.3233/WOR-2012-0537-2876

BUZANELLO, M.R. Influência de variáveis ambientais em frigorífico da unidade climática Subtropical Sul sobre os trabalhadores expostos a baixas temperaturas. 2003. 132 f. Dissertação (Mestrado) - Curso de Engenharia de Produção, Centro Tecnológico, Universidade Tecnológica Federal do Paraná, Florianópolis, 2003.

BUZANELO, M.R.; MORO, A.R.P. Slaughterhouse workers exposed to cold: proposal of reference thermography values for hands. Work, v. 41, n. 1, p. 2876-2881, 2012. http://dx.doi.org/10.3233/WOR-2012-0537-2876.

CASO, M.A.; RAVAIOLI, M.; VENERI, L. Esposizione a sovraccarico biomeccanico degli arti superiori: la valutazione del rischio lavorativo nei macelli avicoli. Prevenzione Oggi, v. 3, n.4, p. 9-21, 2007.

DEJOURS, C.; ABDOUCHELI, E.; JAYET, C. Psicodinâmica do trabalho. São Paulo: Atlas, 1994.

DJAMILA, H.; CHU, C.; KUMARESAN, S. Effect of Humidity on Thermal Comfort in the Humid Tropics. Journal Of Building Construction And Planning Research, v. 2, n. 2 , p.109-117, 2014. http://dx.doi.org/10.4236/jbcpr.2014.22010.

EPSTEIN, Y.; MORAN, D.S. Thermal confort and the heat stress indices. Industrial Health. v. 44, n.3, p. 388- 398, Jul. 2006. https://doi.org/10.2486/indhealth.44.388

FANGER, Povl Ole. Thermal comfort: analysis and applications in environmental engineering. New York: McGraw-Hill Book Company, 1970. 
FROST, P.; ANDERSEN, J.H.; NIELSEN, V.K. Occurrence of carpal tunnel syndrome among slaughterhouse workers. Scand J Work Environ Health, v. 24, n. 4, p. 285-292, ago. 1998. DOI: https://doi.org/10.5271/sjweh.322

GÜTHS, Saulo et al. Body temperature monitoring system for slaughterhouse workers. Advances In Intelligent Systems And Computing, p. 96-105, 11 jun. 2017. http://dx.doi.org/10.1007/978-3-319-60011-6 10.

HASSI, J.; et al. The pituitary-thyroid axis in healthy men living under subarctic climatological conditions. J Endocrinol, v.169, n. 1, p.195-203, 2001. https://doi.org/10.1677/joe.0.1690195

HECK, F.M. Uma Geografia da Degradação do Trabalho: O Adoecimento dos Trabalhadores em Frigoríficos. Revista Percurso. Maringá, v. 5, n. 1, p. 03-31, 2013.

HOLMÉR, I. Cold stress: Part II - The scientific basis (knowledge base) for the guide. Int $\mathbf{J}$ Ind Ergon, v. 14, p. 151-159, 1994. https://doi.org/10.1016/0169-8141(94)90012-4

HOLMÉR, Ingvar. Evaluation of thermal stress in cold regions: a strain assessment strategy. In: INTERNATIONAL SYMPOSIUM ON PROBLEMS WITH COLD WORK, 1., 1997, Solna. [Proceedings...]. Solna: National Institute for Working Life, 1997.

IIDA, I. Ergonomia: projeto e produção. 2. ed. São Paulo: Blucher, 2005. 614 p.

ILMARINEN, R.E.; TAMMELA, E.K. Design of functional work clothing for meat-cutters. Applied Ergonomics, v. 21, n. 1, p. 2-6, 1990. https://doi.org/10.1016/0003-6870(90)90067-8

INSTITUTO BRASILEIRO DE GEOGRAFIA E ESTATÍSTICA (IBGE). Base de dados agregados (SIDRA) Pesquisa trimestral do abate de animais, 2016. Disponível em: http://www.sidra.ibge.gov.br/bda/tabela/listabl.asp?c=1093\&z=t\&o=24. Acesso em: 16 out. 2017.

INTERNATIONAL ORGANIZATION FOR STANDARDIZATION. ISO 7726. Ergonomic of the thermal environment - Instruments for measuring physical quantities. Geneva, Switzerland, 1998.

INTERNATIONAL ORGANIZATION FOR STANDARDIZATION. ISO 7730. Ergonomics of the Thermal Environment - Analytical Determination and Interpretation of Thermal Comfort Using Calculation of the PMV and PPD Indices and Local Thermal Comfort. Geneva Switzerland, 2005.

INTERNATIONAL ORGANIZATION FOR STANDARDIZATION. ISO 8996. Ergonomics of the thermal environment - Determination of metabolic rate. Geneva, Switzerland, 2004.

INTERNATIONAL ORGANIZATION FOR STANDARDIZATION. ISO 9920. Ergonomics of the thermal environment - Estimation of the therm insulation and water vapour resistance of a clothing ensemble. Geneva, Switzerland, 2007.

JAKOBI, H.R.; et al. Benefícios auxílio-doença concedidos aos trabalhadores empregados no ramo de carne e pescado no Brasil em 2008. Cad. Saúde Pública. Rio de Janeiro, v. 31, n. 1, p. 194-207, 2015. https://doi.org/10.1590/0102-311X00087213

JUUL-KRISTENSEN, B.; et al. Physical workload during manual and mechanical deboning of poultry. International Journal Of Industrial Ergonomics, v. 29, n. 2, p.107-115, Fev.

2002. Disponível em: 
http://api.elsevier.com/content/article/PII:S0169814101000518?httpAccept=text/xml. Acesso em: 15 nov. 2017. https://doi.org/10.1016/S0169-8141(01)00051-8

LAMBERTS, R.; XAVIER, A.A.P. Conforto térmico e stress térmico. Laboratório de Eficiência Energética em Edificações. 2012. Disponível em:

http://www.labeee.ufsc.br/publicacoes/relatorios-de-iniciacao-cientifica. Acesso em: 12 abr. 2017.

LEHMUSKALLIO, Eero; HASSI, Juhani; KETTUNEN, Päivi. The skin in the cold. International Journal Of Circumpolar Health, v. 61, n. 3, p. 277-286, set. 2002. http://dx.doi.org/10.3402/ijch.v61i3.17475.

MINISTÉRIO DA AGRICULTURA PECUÁRIA E ABASTECIMENTO. Mapa: aves. 2016. Disponível em: http://www.agricultura.gov.br/animal/especies/aves. Acesso em: 10 set. 2017.

MINISTÉRIO DA PREVIDÊNCIA SOCIAL (INSS). Anuário estatístico de acidentes de trabalho - AEAT. Disponível em: http://www3.dataprev.gov.br/aeat/. Acesso em: 19 set. 2017.

MINISTÉRIO DO TRABALHO E EMPREGO. NR 36: segurança e saúde no trabalho em empresas de abate e processamento de carnes e derivados. 77 ed. São Paulo: Atlas, 2016.

MUSOLIN, K.; et al. Prevalence of carpal tunnel syndrome among employees at a poultry processing plant. Applied Ergonomics, v. 45, n. 6, p.1377-1383, nov. 2014. Disponível em: http://api.elsevier.com/content/article/PII:S0003687014000398?httpAccept=text/xml. Acesso em: 17 nov. 2017. https://doi.org/10.1016/j.apergo.2014.03.005

Nicol, F. A Handbook for field studies toward and adaptive model. Londres: University of East London, 1993.

NICOL, J.f.; HUMPHREYS, M.A. Adaptive thermal comfort and sustainable thermal standards for buildings. Energy And Buildings, v. 34, n. 6, p.563-572, Jul. 2002. http://dx.doi.org/10.1016/s0378-7788(02)00006-3.

NICOL, F.; PARSONS, K. Special issue on thermal comfort standards. Energy And Buildings, [s.I.], v. 34, n. 6, p. 529-532, Jul. 2002. https://doi.org/10.1016/S0378$\underline{7788(02) 00002-6}$

PORTARIA no 210 de 10/11/1998. Dispõe sobre o regulamento técnico da inspeção tecnológica e higiênico-sanitária de carnes de aves. Diário Oficial da União, Brasília, 26 nov. 1998.

REIS, P.F. O trabalho repetitivo em frigorífico: utilização da estesiometria da mão como proposta para avaliação dos níveis de LER/DORT nas síndromes compressivas dos membros superiores. 2012. 137 f. Tese (Doutorado) - Curso de Engenharia de Produção, Centro Tecnológico, Universidade Federal de Santa Catarina, Florianópolis, 2012.

Disponível em: https://repositorio.ufsc.br/bitstream/handle/123456789/100528/308795.pdf?. Acesso em: 20 nov. 2017.

ROHLES, F.H. Thermal Sensations of Sedentary Man in Moderate Temperatures. Human Factors: The Journal of the Human Factors and Ergonomics Society, v. 13, n. 6, p.553560, Dez. 1971. http://dx.doi.org/10.1177/001872087101300606. 
SARDÁ, S.E.; RUIZ, R.S.; KIRTSCHIG, G. A Tutela Jurídica da Saúde dos Empregados de Frigoríficos: Considerações dos Serviços Públicos. Acta Fisiatr, v. 16, n. 2, p. 59-65, 2009.

SOMMERICH, C.M.; MCGLOTHLIN, J.D.; MARRAS, W.S. Occupational risk factors associated with soft tissue disorders of the shoulder: a review of recent investigations in the literature. Ergonomics, [s.l.], v. 36, n. 6, p. 697-717, jun. 1993.

https://doi.org/10.1080/00140139308967931

SUNDSTRUP, E.; et al. Participatory ergonomic intervention versus strength training on chronic pain and work disability in slaughterhouse workers: study protocol for a single-blind, randomized controlled trial. Bmc Musculoskeletal Disorders, v. 14, n. 1, p.67-79, 2013. https://doi.org/10.1186/1471-2474-14-67

SUNDSTRUP, E.; et al. High Intensity Physical Exercise and Pain in the Neck and Upper Limb among Slaughterhouse Workers: Cross-Sectional Study. Hindawi Publishing Corporation: BioMed Research International. New York, p. 1-6, 09 Jan. 2014. Disponível em: http://dx.doi.org/10.1155/2014/218546. Acesso em: 20 mar. 2015. https://doi.org/10.1155/2014/218546

União Brasileira de Avicultura (UBABEF). Exportações brasileiras de carne de frango têm volume recorde para março, diz associação. 2016. Disponível em: http://www.abef.com.br. Acesso em: 10 set. 2017.

XAVIER, A.A.P. Predição de conforto térmico em ambientes internos com atividades sedentárias: teorias física aliada a estudos de campo. 2000. 267 f. Tese (Doutorado) Curso de Engenharia de Produção, Centro Tecnológico, Universidade Federal de Santa Catarina, Florianópolis. Disponível em:

http://www.labeee.ufsc.br/sites/default/files/publicacoes/teses/TESE Antonio Augusto Xavi er.pdf?. Acesso em: 23 ago. 2017.

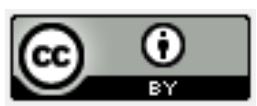

Artigo recebido em: 30/10/2018 e aceito para publicação em: 01/07/2021 DOI: http://dx.doi.org/10.14488/1676-1901.v21i2.3424 